\title{
Surgical Outcome of Children and Adolescents with Tethered Cord Syndrome
}

\author{
Toshitaka Seki ${ }^{1}$, Kazutoshi Hida ${ }^{2}$, Shunsuke Yano ${ }^{2}$, Toru Sasamori ${ }^{1}$, \\ Shuji Hamauch ${ }^{1}$, Izumi Koyanagi ${ }^{3}$, Kiyohiro Houkin ${ }^{1}$ \\ ${ }^{I}$ Department of Neurosurgery, Hokkaido University Graduate School of Medicine, Sapporo, Japan \\ ${ }^{2}$ Department of Neurosurgery, Sapporo Azabu Neurosurgical Hospital, Sapporo, Japan \\ ${ }^{3}$ Department of Neurosurgery, Hokkaido Neurosurgical Memorial Hospital, Sapporo, Japan
}

\section{Study Design: Retrospective cohort study.}

Purpose: To compare long-term results of surgery with the outcomes of symptomatic and asymptomatic tethered cord syndrome (TCS) in children and adolescents and to assess the surgery duration for those with TCS.

Overview of Literature: Pediatric patients with TCS continue to pose significant diagnostic and management challenges.

Methods: We retrospectively analyzed the outcomes of 31 patients (16 males, 15 females) with TCS, including 21 with lumbosacral lipoma. All were surgically treated between 1989 and 2015. They were divided into symptomatic and asymptomatic TCS groups. The results of the treatment were summarized and analyzed using a non-parametric Mann-Whitney $U$ test.

Results: Nineteen patients had symptomatic TCS and 12 had asymptomatic TCS. Patients had a median age of 34 months (range, 0-201 months). The median follow-up period was 116 months (range, 7-223 months). Of the 19 symptomatic TCS patients, preoperative deficits improved after surgery in two (10.5\%) and remained stable in $17(89.5 \%)$ patients. One of the 12 asymptomatic TCS $(8.3 \%)$ patients showed an exacerbated illness after surgery, and one in 11 patients remained stable (11.7\%). There were significant differences in monthly age at surgery, preoperative bowel and bladder dysfunction, neurological function, presence/absence of clean intermittent catheterization introduction, and presence/absence of motor disorder at final follow-up (all $p<0.05$ ). In the univariate analysis, the presence/absence of preoperative bowel and bladder dysfunction, and symptoms were strongly associated with the risk of children and adolescents with TCS $(p<0.05)$.

Conclusions: Early accurate diagnosis and adequate surgical release might lead to successful outcomes in children and adolescents with TCS. Surgical untethering is a safe and effective method for treatment of children with TCS.

Keywords: Children; Lumbosacral; Lipoma; Spina bifida; Outcome

\section{Introduction}

Tethered cord syndrome (TCS) comprises a combination of neurological deficits in the lower limbs, bowel and bladder disturbances, and orthopedic deformities, which are usually diagnosed in childhood [1,2]. The natural history of cord tethering remains unclear, but not all children experience deterioration. In TCS, progressive neurological deterioration in the functions of the lower spinal cord results from traction on the conus medullaris [3].

Received Feb 16, 2016; Revised Mar 15, 2016; Accepted Mar 22, 2016

Corresponding author: Toshitaka Seki

Department of Neurosurgery, Hokkaido University Graduate School of Medicine,

N-15, W-7, Kita-ku, Sapporo 0608638, Japan

Tel: +81-11-706-5987, Fax: +81-11-708-7737, E-mail: toseki1@hotmail.com 
Although the symptoms are variable, tethered conus should be suspected in patients with an unexpected spastic gait, neurogenic bladder, bowel dysfunction, lower extremity weakness, scoliosis, or foot deformities [3-5]. The pathophysiology of TCS is unclear and its treatment is mainly surgical. Untethering the spinal cord is essential for effective improvement in TCS [2]. Cutting the filum terminal is crucial for a satisfactory untethering procedure [6]. The treatment is mainly surgical untethering in children with TCS [2]. However, the timing of surgical treatment for TCS patients is still controversial.

The aim of this study is to present a series of children and adolescents with TCS and to document clinical characteristics of this syndrome. Furthermore, we considered the timing of surgery for asymptomatic TCS.

\section{Materials and Methods}

From 1989 to 2015, 31 children and adolescents underwent surgical treatment for TCS in our department. The data from these 31 patients were reviewed retrospectively. All had symptomatic or asymptomatic TCS. They were examined for their clinical features and for their surgical outcomes from this syndrome. The children and adolescents with TCS were either neurologically intact or had a variety of neurological deficits. Preoperative and postoperative neurological function was assessed, and a functional grade was assigned using a previously published classification scheme (Table 1) [7].

For symptomatic and asymptomatic comparison of TCS cases, we compared the age in months at surgery, presence/absence of lipoma or myelomeningocele, motor deficit, sensory disturbance, bowel and bladder dysfunction, clean intermittent catheterization introduction, and functional grading at final follow-up using the Mann-Whitney
$\mathrm{U}$ test. We defined grade 0 as a good-prognosis group and grades 2 and 3 as a poor-prognosis group.

Univariate analysis was performed to determine the impact of various patients and treatment variables on TCS. Multivariate Cox proportional hazard models were used to calculate hazard ratios (HRs) and $95 \%$ confidence intervals (CIs) were generated for investigation of independent predictors of prognosis in children and adolescents with TCS. All variables were assessed in a stepwise multivariate model that required a $p<0.1$ significance for variable entry and retention into the model; $p<0.05$ was considered statistically significant. All analysis was performed using Ekuseru-Toukei 2015 software (OMS Ltd., Tokorozawa, Japan).

\section{Results}

All 31 patients underwent surgery for release of the tethered conus. Sixteen (51.6\%) patients were male and 15 were female. Symptomatic TCS was detected in 19 patients (61.3\%) and asymptomatic TCS in 12 . The median duration of follow-up was 116 months (range, 7-223 months) overall, and was 68 months (range, 8-221 months) and 116 months (range, 7-223 months) in patients with symptomatic and asymptomatic TCS, respectively. Lumbosacral lipoma was associated with a lesion in 21 patients (67.7\%) and meningomyelocele in eight patients (25.8\%). Lipoma was caudal in 10 patients, transitional in four, filar in three, lipomeningocele in three, and unknown in one case. Preoperative functional grading was Grade 0 in 12 patients, Grade 2 in 13, and Grade 3 in six patients. No patient was Grade 1, 4, or 5. Functional grading at the final follow-up was Grade 0 in 14 patients, Grade 2 in 11, and Grade 3 in six. All asymptomatic TCS patients were Grade 0 at final follow-up. There was a significant differ-

Table 1. Function grading for patients with tethered cord syndrome

\begin{tabular}{ll}
\hline Grade 0 & No significant neurological, orthopedic, or urological problem. \\
Grade 1 & The patients rnay have reflex changes and/or sensory deficits. \\
Grade 2 & $\begin{array}{l}\text { Minimal weakness and/or muscle wasting and/or foot deformity affecting only one leg without significant gait } \\
\text { disturbance. Norrnal bladder andsphincter function. }\end{array}$ \\
Grade 3 & $\begin{array}{l}\text { Meakness affecting both legs. } \\
\text { weakness of both legs combined with neurogenic bladder. }\end{array}$ \\
Grade 4 & Severe paraparesis requiring aids for walking, with or without neurogenic bladder. \\
Grade 5 & Inability to ambulate.
\end{tabular}


ence at the final follow-up between the symptomatic TCS group and the asymptomatic TCS group $(p<0.01)$.

The spinal level of the conus lipoma was determined from magnetic resonance imaging (MRI) in all patients with TCS. The number of patients with conus medullaris was 12 in the lumbar spine region (38.7\%), one in the L5S1 level (3.2\%), and 18 in the sacral spine region (58.1\%).

Median age of months at surgery was 34 months (range, 0-201 months) overall, 58 months (range, 5-201 months) in patients with symptomatic TCS, and 32 months (range, 0-66 months) in patients with asymptomatic TCS (Fig. 1). There was a significant difference between the two groups $(p=0.045)$.

Eighteen (58.1\%) and $16(51.6 \%)$ patients had bowel and bladder dysfunction preoperatively and at final follow-up, respectively. All TCS patients had a medical examination by urologists before and after surgery. Of the patients with asymptomatic TCS, only one patient exhibited lipoma (transitional type) at final follow-up. Three patients with symptomatic TCS showed improvement of symptoms in the final follow-up. There was a significant difference between the two groups at final follow-up $(p<0.01)$. Furthermore, 9 patients with symptomatic TCS and one patient with asymptomatic TCS had received a clean intermittent catheterization. Similarly, there was a significant difference between the groups at final followup $(p=0.026)$.

Seven patients (22.6\%) had motor deficits preoperatively. Motor deficit was still present in these patients at the final follow-up. No asymptomatic TCS patients displayed motor deficit at the final follow-up. There was a significant difference between the two groups ( $p=0.019$ ).

In addition, six patients (19.4\%) showed a sensory disturbance preoperatively. At final follow-up, sensory disturbance was observed in seven patients. For patients with asymptomatic TCS, two patients had sensory disturbance at final follow-up. However, there was no significant difference between the two groups ( $p=0.44$ ).

The univariate analysis of factors associated with prognosis revealed that asymptomatic TCS was significantly related to good prognosis (HR, 58.7; 95\% CI, 5.4-640.1; $p<0.001$ ), while preoperative bowel and bladder dysfunction was associated with a significantly poor prognosis (HR, 27.5; 95\% CI, 3.9-193.5; $p<0.001$ ). However, age at surgery approached, but was not significantly associated with prognosis in children and adolescents with TCS (HR, 4.6; 95\% CI, 0.995-21.1; $p=0.051)$. However, there were no significant variables in the multivariate stepwise analysis (data not shown).

\section{Discussion}

Huang at al. [8] performed surgical treatment for 25 children including asymptomatic lipomeningocele in 20 patients. These 20 patients remained symptom-free and three of five symptomatic patients displayed resolution or improvement in symptoms. The authors concluded that surgery for lipomeningocele should be performed within

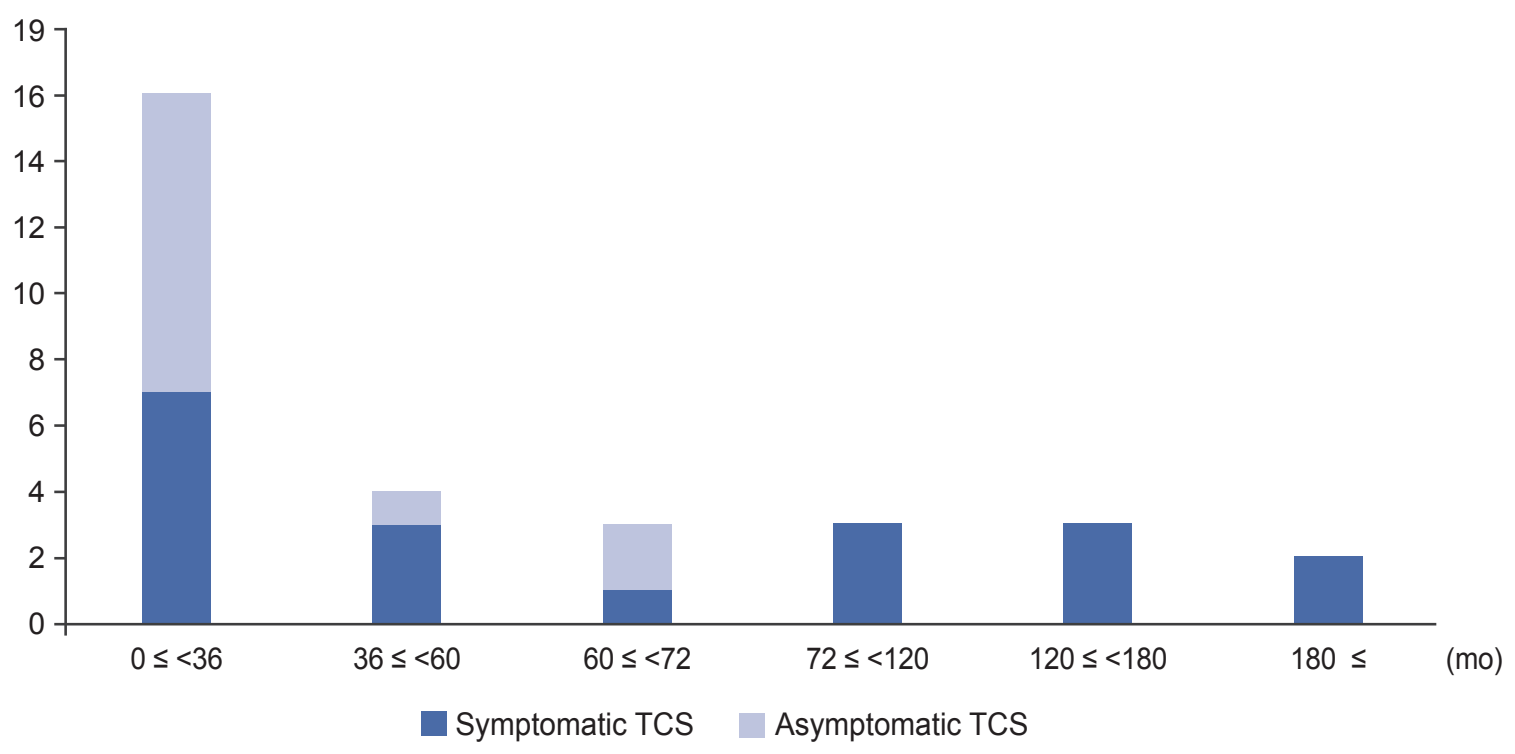

Fig. 1. Age distribution in 31 patients with tethered cord syndrome (TCS). 
3-6 months of birth, or at the earliest possible time after this age. Kulkarni et al. [9] reported on the conservative management of 53 children with asymptomatic spinal lipomas of the conus. In a comparison with patients who underwent early surgery, there was no significant difference in the risk of neurological deterioration over a follow-up period of nine years.

In the present study, the overall median age at surgery was 34 months; patients with asymptomatic TCS were significantly younger than patients with symptomatic TCS (median age of months at surgery; 32 vs. 58 months). We performed univariate analysis as an objective variable for prognosis, and as an explanatory variable for those patients more than 35 months and less than 35 months of age. Although no significant difference was evident, the patients who underwent surgery at $<35$ months of age tended had a good prognosis $(p=0.051)$. Additionally, in the final follow-up, the patients with asymptomatic TCS maintained symptom-free or had only slight neurological dysfunction. The results indicate that children with asymptomatic TCS benefit from prophylactic surgery performed by 34 months of age, or at their earliest opportunity after this age. However, the age of untethering was not an independent predictor of prognosis.

In this study, although there were no obvious abnormal findings, such as tethering or regrowth of lipoma, as evidenced by MRI during follow-up, one patient with asymptomatic TCS showed tardive bowel and bladder dysfunction at final follow-up. Concerning tardive neurological disorder of TCS, Pierre-Kahn et al. [10] reported that 15 of 32 patients (46.9\%) with asymptomatic lumbosacral lipoma developed tardive neurological disorder after surgery during a follow-up period of more than 5 years. Furthermore, Xenos et al. [11] performed surgery in 59 children with lumbosacral lipomas, including 19 patients with asymptomatic TCS. During a follow-up period, five patients (26\%) with asymptomatic TCS developed bowel and bladder dysfunction. The authors concluded that prophylactic surgery for patients with asymptomatic TCS affords some protection for future neurological deterioration.

Reports on the natural history of asymptomatic TCS have shown that deterioration occurs at a proportion of about $3 \%-4 \%$ per year $[9,12,13]$. Wykes et al. [12] described the cumulative risk of deterioration with asymptomatic lumbosacral lipoma was $40 \%$ at 10 years. Furthermore, female children $<2$ years of age with tran- sitional type of lumbosacral lipomas and associated with syrinx of the spinal cord were at high risk of deterioration. Talamonti et al. [13] performed prophylactic surgery or conservative treatment in 56 patients with asymptomatic lipoma of the conus medullaris. Symptoms of TCS occurred in $9.7 \%$ and $29.1 \%$ of surgically treated and conservatively managed patients, respectively. Although no significant difference was noted, a clear trend for surgery was indicated $(p=0.067)$. On the other hand, in a prospective study on patients with asymptomatic lipoma of the conus involving comparison with patients who underwent prophylactic surgery previously, no difference between either group was reported [9].

In the present study, one of 12 patients with asymptomatic TCS (8.3\%) developed tardive neurological disorder after prophylactic surgery during a follow-up period of more than 9 years (mean, 105 months; median, 116 months). Although this series is small, we believe that prophylactic surgery for asymptomatic TCS is adequate in preventing deterioration of neurological function.

There are some limitations to this study. First, the sample size of children and adolescent with TCS was small, which makes it difficult to truly assess the differences between the natural history of TCS and prophylactic surgery for patients with asymptomatic TCS. Second, only 13 of the remaining 31 patients were followed up for $<5$ years at the time of this study. We intend to continue to monitor this cohort of patients, especially as many of these infants and young children progress to adolescence.

\section{Conclusions}

We report 31 patients with TCS, including 12 patients with asymptomatic TCS who underwent prophylactic surgery. In our study, patients who underwent prophylactic surgery for asymptomatic TCS showed a significant improvement in neurological prognosis. We conclude that prophylactic surgery for TCS should be conducted in those aged $<34$ months, or as soon as possible. Careful follow-up should be carried out to permit prompt reintervention in cases that deteriorate.

\section{Conflict of Interest}

No potential conflict of interest relevant to this article was reported. 


\section{References}

1. Kang JK, Yoon KJ, Ha SS, Lee IW, Jeun SS, Kang SG. Surgical management and outcome of tethered cord syndrome in school-aged children, adolescents, and young adults. J Korean Neurosurg Soc 2009;46:46871.

2. Solmaz I, Izci Y, Albayrak B, et al. Tethered cord syndrome in childhood: special emphasis on the surgical technique and review of the literature with our experience. Turk Neurosurg 2011;21:516-21.

3. Fitz CR, Harwood Nash DC. The tethered conus. Am J Roentgenol Radium Ther Nucl Med 1975;125:51523.

4. Lee GY, Paradiso G, Tator CH, Gentili F, Massicotte EM, Fehlings MG. Surgical management of tethered cord syndrome in adults: indications, techniques, and long-term outcomes in 60 patients. J Neurosurg Spine 2006;4:123-31.

5. Steinbok P, Garton HJ, Gupta N. Occult tethered cord syndrome: a survey of practice patterns. J Neurosurg 2006;104(5 Suppl):309-13.

6. Kural C, Solmaz Y, Pusat S, et al. Surgical technique for split cord halformations: pitfalls and solution pathways. Gulhane Med J 2013;55:77-83.

7. Hoffman HJ, Taecholarn C, Hendrick EB, Humphreys RP. Management of lipomyelomeningoceles. Experience at the Hospital for Sick Children, Toronto. J Neurosurg 1985;62:1-8.

8. Huang SL, Shi W, Zhang LG. Surgical treatment for lipomyelomeningocele in children. World J Pediatr 2010;6:361-5.

9. Kulkarni AV, Pierre-Kahn A, Zerah M. Conservative management of asymptomatic spinal lipomas of the conus. Neurosurgery 2004;54:868-73.

10. Pierre-Kahn A, Zerah M, Renier D, et al. Congenital lumbosacral lipomas. Childs Nerv Syst 1997;13:298334.

11. Xenos C, Sgouros S, Walsh R, Hockley A. Spinal lipomas in children. Pediatr Neurosurg 2000;32:295-307.

12. Wykes V, Desai D, Thompson DN. Asymptomatic lumbosacral lipomas: a natural history study. Childs Nerv Syst 2012;28:1731-9.

13. Talamonti G, D’Aliberti G, Nichelatti M, Debernardi A, Picano M, Redaelli T. Asymptomatic lipomas of the medullary conus: surgical treatment versus conservative management. J Neurosurg Pediatr 2014;14: 245-54. 